Bull. Austral. Math. Soc.

05в05

VoL. 41 (1990) [97-112]

\title{
ON THE MINIMUM NUMBER OF BLOCKS DEFINING A DESIGN
}

\author{
Ken GraY
}

\begin{abstract}
A set of blocks which is a subset of a unique $t-\left(v, k, \lambda_{t}\right)$ design is said to be a defining set of that design. We examine the properties of such a set, and show that its automorphism group is related to that of the whole design. Smallest defining sets are found for 2-designs and 3-designs on seven or eight varieties with block size three or four, revealing interesting combinatorial structures.
\end{abstract}

\section{DEFINING SETS: DEFINITIONS AND BASIC RESULTS}

A design is a collection of $b$-subsets (blocks) chosen from a set of $v$ elements, $V$ say. A block design is a collection of blocks chosen in such a way that every element belongs to $r$ blocks. If $k<v$, we say the block design is incomplete. Except in Section 4, where linked designs are dealt with, we will take all designs to be incomplete block designs. If every subset of $t$ elements belongs to exactly $\lambda_{t}$ blocks for some constant $\lambda_{t}$, we call the design a $t$-design and indicate its parameters by $t-\left(v, k, \lambda_{t}\right)$. When $t=2$, we say the design is balanced. We shall often omit the subscript, and simply write $t-(v, k, \lambda)$.

A set of blocks which is a subset of a unique $t-\left(v, k, \lambda_{t}\right)$ design is said to be a defining set of the design, and will be denoted by $d\left(t-\left(v, k, \lambda_{t}\right)\right)$. (This notation later requires us to denote the index of the $i$ th design by $\lambda(i)$, rather than by $\lambda_{i}$.) For example, the set of blocks $R=\{123,145,167\}$ can be completed to a $2-(7,3,1)$ design in two distinct ways: by adjoining either $T_{1}=\{246,257,347,356\}$ or $T_{2}=$ $\{247,256,346,357\}$. Hence $R$ is not a defining set of either design. But the set of blocks $S=\{123,145,246\}$ can be completed to a $2-(7,3,1)$ design in only one way, namely by adjoining the blocks $\{167,257,347,356\}$. Hence $S$ is a defining set of that design.

A minimal defining set, denoted by $d_{m}(t-(v, k, \lambda))$, is a defining set, no proper subset of which is a defining set. A smallest defining set, denoted by $d_{s}(t-(v, k, \lambda))$, is a defining set such that no other defining set has smaller cardinality. Every $t$-design clearly has a defining set (the whole design) and hence a smallest defining set. A $d(t-(v, k, \lambda))$ defining set consisting of blocks of a particular $t-(v, k, \lambda)$ design $D$ is abbreviated to $d D$.

Received 17th February 1989

Copyright Clearance Centre, Inc. Serial-fee code: 0004-9729/90 \$A2.00+0.00. 
In this section, we establish several lemmas.

We define a collection of blocks $\mathcal{T}_{1}$ to be a trade if there exists a distinct collection $T_{2}$ containing precisely the same pairs; for example, the collections $T_{1}$ and $T_{2}$ given above. Others use the term similarly to mean the set $\left\{\mathcal{T}_{1}, \mathcal{T}_{2}\right\}$ of distinct collections containing precisely the same pairs (see Billington [2] and Gray [5]). Such collections are also known as mutually balanced (Rodger [10]).

When forming unions of sets of blocks we take the symbol $U$ as allowing repeated blocks; for example

$$
\{123,456\} \cup\{123,789\}=\{123,123,456,789\} .
$$

We also denote the complement of the set $X$ by $\tilde{X}$ and $X \cap \tilde{Y}$ by $X \backslash Y$.

LEMMA 1.1. Every defining set $S$ of a $2-(v, k, \lambda)$ design $D$ contains a block of every possible trade $T_{1} \subseteq D$.

Proof: Suppose $T_{1} \subseteq D$ has a trade $T_{2}$ and $S \cap T_{1}=\emptyset$. Then $S \subseteq D \backslash T_{1}$ and the designs $T_{i} \cup\left(D \backslash T_{1}\right)$, for $i=1,2$, are a pair of distinct $2-(v, k, \lambda)$ designs containing $S$, contradicting the assumption that $S$ is a defining set.

Now every permutation on the elements of $V$ induces a mapping from one $k$-set to another. An automorphism of a set of blocks $X$ is a permutation of the elements which takes every block of $X$ to a block of $X$. Let $A u t(X)$ denote the group of all the automorphisms.

Lemma 1.2. Suppose $S$ is a particular defining set of a $t-\left(v, k, \lambda_{t}\right)$ design $D$ and $\rho \in A u t(D)$. Then $\rho(S)$ is also a defining set of $D$ and $A u t(S)$ is a subgroup of $\operatorname{Aut}(D)$.

Proof: Suppose $\rho$ is an automorphism of $D$, that is, $\rho(D)=D$, where $\rho$ is a permutation on the elements of the underlying $v$-set. Clearly, if $S$ is a defining set of $D$ then $\rho(S)$ is also a defining set of $D$.

Further suppose that $\rho^{*}$ is any automorphism of $S$. Since $S \subseteq D$ then we have $\rho^{*}(S) \subseteq \rho^{*}(D)$. So $S=\rho^{*}(S)$ is a subset of the designs $D$ and $\rho^{*}(D)$. But, if $D$ is a $t-\left(v, k, \lambda_{t}\right)$ design, then so is $\rho^{*}(D)$ and, since $S$ is a defining set, $D=\rho^{*}(D)$. Hence $\rho^{*}$ is an automorphism of $D$.

LEмма 1.3. No automorphism of a $2-(v, k, 1)$ design, with $k>2$, consists of a single transposition.

Proof: Without loss of generality, suppose we have such a $2-(v, k, 1)$ design on $\{1,2, \ldots, v\}$, and that this design is fixed under the permutation (12). Since the design is incomplete, the element 1 belongs to at least two blocks, say $b_{1}=\left\{1,2, a_{3}, \ldots, a_{k}\right\}$ and $b_{2}=\left\{1, c_{2}, c_{3}, \ldots, c_{k}\right\}$, where $2 \notin b_{2}$. Since (12) is an automorphism, $b_{2}$ and $(1,2) \mathrm{b}_{2}$ are distinct blocks containing the pair $\left\{c_{2}, c_{3}\right\}$, contradicting the fact $\lambda=1$. 0 
Lemma 1.4. Any $d(2-(v, k, 1))$ defining set $S$, for $k>2$, has at least $(v-1)$ elements occurring in its blocks.

Proof: If two elements $i$ and $j$ do not appear in any block of $S$, then we have $(i, j) \in A u t(S)$. By Lemma 1.2, $(i, j) \in A u t(D)$ where $D$ is the unique $2-(v, k, 1)$ design containing $S$, contradicting Lemma 1.3 .

LEMMA 1.5. Suppose two elements $i, j$ each appear only once in a $d(2-(v, k, 1))$ defining set $S$, where $k>2$. Then $i, j$ cannot appear in the same block of $S$.

Proof: Suppose $i, j$ appear uniquely in the same block of $S$. Then $(i, j) \in \operatorname{Aut}(S)$ and again we have a contradiction of Lemma 1.3 .

These lemmas lead to a simple lower bound on the number of blocks in a defining set of a $2-(v, k, 1)$ design.

Theorem 1.6. For every $2-(v, k, 1)$ design $D$, with $k>2$,

$$
|d D| \geqslant \frac{2(v-1)}{k+1} \text {. }
$$

Proof: Let $S$ be a defining set of $D$, and suppose that $|S|=s$. Then by Lemma 1.5 , at most $s$ elements occur in precisely one block each of $S$, and by Lemma 1.4, at least $(v-1-s)$ elements occur in at least two blocks each of $S$. Since we have only $k s$ entries in the blocks, we need

$$
s+2(v-1-s) \leqslant k s
$$

and the result follows.

Were one to consider the effect on the bound of changing the value of $\lambda$, it would be worth observing that a defining set may have cardinality 0 . This is true, for example, of a $d_{s}(2-(4,3,2))$.

The following Lemmas are given without proof.

LEMмA 1.7. Suppose $D=\left\{b_{i}\right\}$ is a block design. Then the set of complementary blocks $\widetilde{D}=\left\{\widetilde{b}_{i}\right\}$, where complementation is with respect to the set of varieties $V$, is also a block design (see [12]).

LEMMA 1.8. If $S$ is a particular defining set of a design $D$, then $\widetilde{S}$ is a defining set of $\tilde{D}$.

A $t-\left(v, k, \lambda_{t}\right)$ design $D$ is said to be reducible if $D=D_{1} \cup D_{2}$ for some $t-\left(v, k, \mu_{t}\right)$ and $t-\left(v, k, \omega_{t}\right)$ designs $D_{1}$ and $D_{2}$ respectively.

TheOREM 1.9. Suppose that $S$ is a defining set of some reducible $t-\left(v, k, \omega_{t}+\mu_{t}\right.$, design $D=D_{1} \cup D_{2}$, where $D_{1}$ and $D_{2}$ are $t-\left(v, k, \omega_{t}\right)$ and $t-\left(v, k, \mu_{t}\right)$ designs respectively. Then

$$
|S| \geqslant\left|d_{s} D_{1}\right|+\left|d, D_{2}\right|
$$


Proof: Suppose $|S|<\left|d_{s} D_{1}\right|+\left|d_{s} D_{2}\right|$. For the partition of the blocks of $D$ into blocks of $D_{1}$ and blocks of $D_{2}$, we partition the blocks of $S$ correspondingly with $n_{j}$ blocks in $D_{j}$, for $j=1,2$. Then $n_{j}<\left|d_{s} D_{j}\right|$ for at least one value of $j=1$ or 2 , and for this value of $j$ these $n_{j}$ blocks belong to two distinct designs $\mathcal{D}_{1}$ and $\mathcal{D}_{2}$ say, with the same parameters as $D_{j}$. So $S$ is a subset of two distinct $t-\left(v, k, \omega_{t}\right)$ designs, namely $D_{l} \cup \mathcal{D}_{1}$ and $D_{l} \cup \mathcal{D}_{2}$, where $l=1$ or 2 and $l \neq j$. Hence $S$ cannot be a defining set.

Corollary 1.10. If each $D(i)$ is a $2-(v, k, \omega(i))$ design for $i=1, \ldots, n$ and if $D=\bigcup_{i=1}^{n} D(i)$ is a union of $2-(v, k, \omega(i))$ designs, then

$$
\left|d_{s} D\right| \geqslant \sum_{i=1}^{n}\left|d_{s} D(i)\right|
$$

\section{Properties of SOME SMall DesignS}

In this section, we list the properties of some small designs which we shall use in constructing examples, and give their smallest defining sets.

Example 2.1. The Fano Plane.

There is, up to isomorphism, just one $2-(7,3,1)$ design, also known as the Fano plane. It has automorphism group of order 168 (Biggs and White [1]) and hence there are $7 ! / 168=30$ possible planes based on the set of seven elements; for example, $R \cup T_{1}$ as given in Section 1. As indicated there, it is easily verified that any three blocks with no element common to all three form a defining set; and that trades can be established by completing any three blocks containing a cómmon element in the two possible ways. Since no three blocks containing a common element form a defining set, neither will any set of fewer than three blocks. Thus we have:

THEOREM 2.2.- The smallest defining sets of a Fano plane are the sets of three blocks containing no element common to all three.

Example 2.3. The Four $2-(7,3,2)$ Designs.

There are, up to isomorphism, four $2-(7,3,2)$ designs (see Stanton and Collens [11], Wallis [13] and Nandi [9]). Examples of these are given in Table 1 and labelled $A, B, C$ and $D$. All are reducible, with Table 1 expressing each design as the union of two permutations of the Fano plane $P$ with blocks

$$
123145167246257347356 \text {. }
$$

For convenience we let $P^{1}=(2765) P, P^{2}=(274) P, P^{3}=(142) P$ and $P^{4}=(347) P$. 
Table 1. The Smallest Defining Sets of the Non-isomorphic $2-(7,3,2)$ Designs.

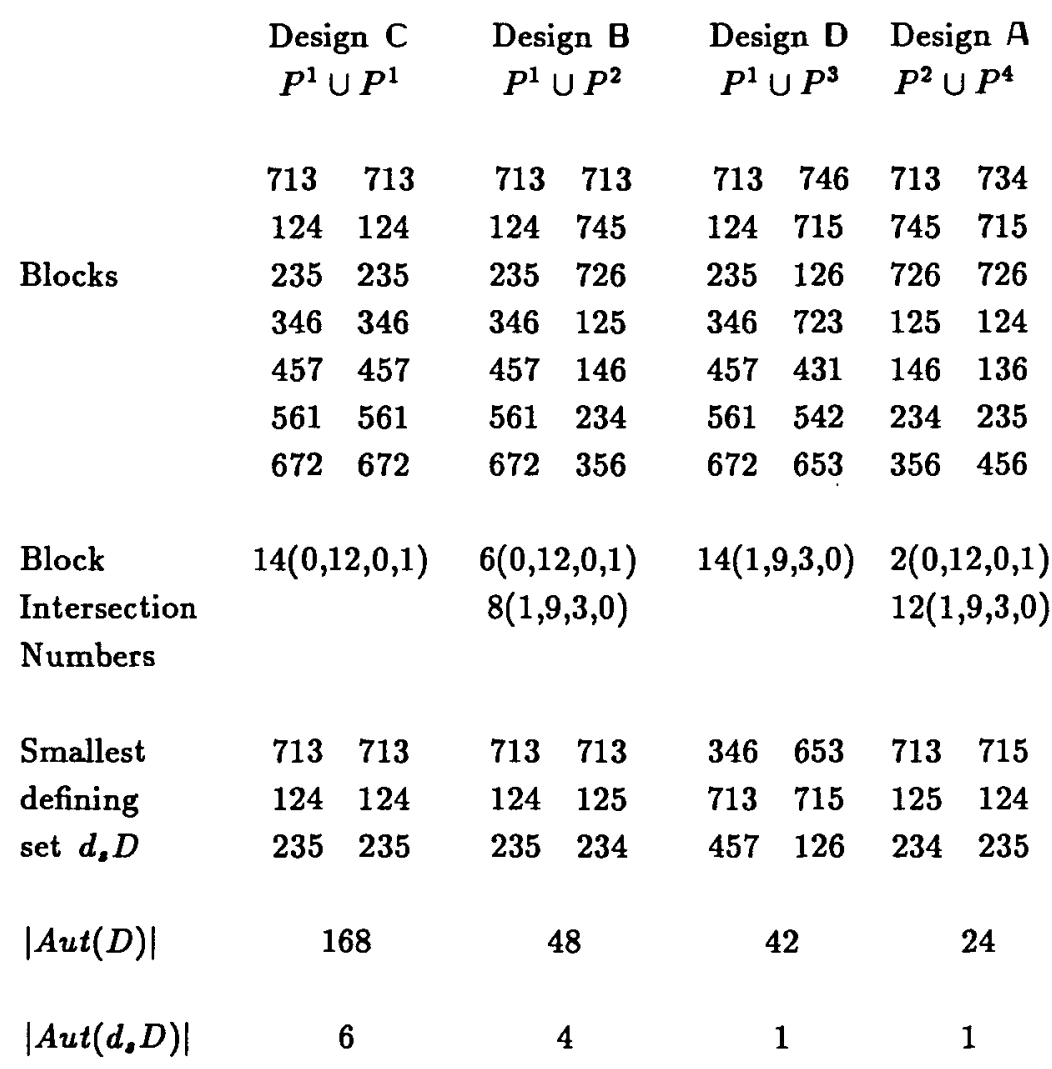

For a particular block b of a given $2-(v, k, \lambda)$ design let $a_{i}$ be the number of blocks intersecting $b$ in exactly $i$ elements. The numbers $a_{i}$ are called block intersection numbers and a block said to be of type $\mathbf{a}=\left(a_{0}, a_{1}, \ldots, a_{k}\right)$ if it has these block intersection numbers. If a design has $\alpha$ blocks of type $\left(a_{0}, a_{1}, \ldots, a_{k}\right)$, we write $\alpha\left(a_{0}, a_{1}, \ldots, a_{k}\right)$ or $\alpha \mathbf{a}$. For $2-(7,3,2)$ designs, blocks are of types $\mathbf{a}$ or $\mathbf{b}$ where $\mathbf{a}=(0,12,0,1), \mathbf{b}=(1,9,3,0)$. The number of blocks of each type of each design are given in Table 1, establishing the non-isomorphism of the designs. Table 1 also gives a smallest defining set for each design, together with the order of the automorphism group of the defining set.

To check that each given smallest defining set is, in fact, a defining set we use the properties that any two blocks of a Fano plane intersect in one element and that any $2-(7,3,2)$ design is reducible, together with Theorem 2.2. For example, if the blocks $713,713,124,125,234,235$ belong to a $2-(7,3,2)$ design $D=D_{1} \cup D_{2}$, where $D_{1}$ and $D_{2}$ are Fano planes, then each Fano plane contains 713, and one contains 124 and the 
other 125. The design containing 125 cannot contain 235 and hence must contain 234 . Then we have that $D_{1}$, say, contains 713,124 and 235 while $D_{2}$ contains 713,125 and 234. In each case we have three blocks with no common element, forming a defining set of a Fano plane, and thus $D$ is uniquely determined. That we have a smallest defining set is easily seen from Theorem 1.9 The other smallest defining sets given in Table 1 can be similarly verified. Hence we have

TheOREM 2.4. Every $2-(7,3,2)$ design has a smallest defining set consisting of six blocks.

The number of such defining sets which are distinct up to isomorphism is not considered in this paper.

\section{SMALlest Defining SETS of $2-(7,3,3)$ DESIGNS}

Morgan [8] showed that there are ten non-isomorphic $2-(7,3,3)$ designs, only one of which is irreducible. Since no $2-(7,3,2)$ design is irreducible, each of the reducible $2-(7,3,3)$ designs can be partitioned into three Fano planes. Hence, by Corollary 1.10, defining sets of the reducible $2-(7,3,3)$ designs contain at least nine blocks.

ThEOREM 3.1. Every reducible $2-(7,3,3)$ design has a smallest defining set of nine blocks.

Proof: As given in Example 2.3, let $\mathrm{P}$ be the Fano plane with blocks $\{123,145,167,246,257,347,356$,$\} .$

Then Table 2 gives the nine reducible $2-(7,3,3)$ designs, numbered $1,2, \ldots, 9$, as in [8], and a defining set of nine blocks partitioned into the possible three sets of three blocks with any pair of blocks intersecting in one element. It is easily seen that each set of three blocks is a defining set of a Fano plane.

Note that design number 5 has two possible partitions of the nine blocks, corresponding to two possible partitions into Fano planes.

In each of the nine designs the block 123 is repeated. As will be seen later, the existence of a repeated block in the defining set ensures each design is reducible in this case. The order of each automorphism group is also given. 
Table 2. The Smallest Defining Sets of the Nine Reducible 2-(7,3,3) Designs.

\# Design D

$1 \quad P \cup P \cup P$

$2 \quad P \cup P \cup(12) P$

$3 \quad P \cup(12) P \cup(14) P$

$4 \quad P \cup P \cup(567) P$

$5 \quad P \cup(567) P \cup(576) P$ (67) $P \cup(56) P \cup(57) P$

$6 \quad P \cup P \cup(3456) P$

$7 \quad P \cup(45) P \cup(346) P$

$8 \quad P \cup(567) P \cup(34)(67) P$

$9 \quad P \cup(567) P \cup(3467) P$
Defining set dD

$123,356,246 ; 123,356,246 ; 123,356,246$

$123,356,257 ; 123,356,257 ; 123,356,157$

$123,356,246 ; 356,123,425 ; 356,423,145$

$123,145,246 ; 123,145,246 ; 123,146,345$

$123,257,437 ; 123,256,435 ; 123,267,436$

$123,256,436 ; 123,267,437 ; 123,257,435$

$123,246,347 ; 123,246,347 ; 124,267,346$

$123,145,356 ; 123,145,256 ; 124,156,236$

$123,145,356 ; 123,345,146 ; 124,135,237$

$123,145,246 ; 123,146,247 ; 124,235,137$

Order of each automorphism group

$\begin{array}{lccccccccc}\text { Design \# } & 1 & 2 & 3 & 4 & 5 & 6 & 7 & 8 & 9 \\ |\operatorname{Aut}(D)| & 168 & 24 & 8 & 12 & 144 & 21 & 3 & 6 & 6 \\ |\operatorname{Aut}(S)| & 4 & 1 & 1 & 1 & 6 & 1 & 1 & 1 & 2\end{array}$

We now consider smallest defining sets of the unique irreducible $2-(7,3,3)$ design.

LEмма 3.2. The unique irreducible $2-(7,3,3)$ design has a defining set of seven blocks.

Proof: The 21 blocks of the irreducible $2-(7,3,3)$ design on $\{1,2, \ldots, 7\}$ can be obtained by taking the $\left(\begin{array}{l}7 \\ 3\end{array}\right)=35$ possible triples and deleting the blocks of any two disjoint Fano planes (see Street and Street [12]). We choose to delete the Fano planes obtained by cycling starter blocks 124 and 134 modulo 7 . This leaves the design consisting of all the triples in arithmetic progression modulo 7 , which is type 10 in [8], and which we call $I$.

Consider the set of blocks $S_{1}=\{123,125,456,145,127,135,345\}$. Now $S_{1} \subset I$. Consideration of block intersection sizes ensures this set of seven blocks cannot be partitioned into three subsets of Fano planes, and hence can only belong to a $2-(7,3,3)$ design of type 10, containing no repeated blocks.

Suppose $S_{1} \subset D$, where $D$ is a $2-(7,3,3)$ design. There can be no further occurrences of pairs 12,45 or 15 so we have additional blocks

$$
14^{*} 14^{*} 1^{* *} 1^{* *} 24^{*} 24^{*} 24^{*} 25^{*} 25^{*} 2^{* *} \quad 4^{* *} 5^{* *} 5^{* *} 367,
$$


where asterisks indicate elements still to be chosen. Now the blocks containing 24 cannot be 241 or 245 and there are no repeated blocks. Also, 47 must occur twice more, leading to

$$
14714^{*} 1^{* *} 1^{* *} \quad 24324624725^{*} 25^{*} 2^{* *} \quad 47^{*} 5^{* *} 5^{* *} 367
$$

Here 57 has not occurred, and 36 has only occurred once, so to avoid block repetition we have

\section{$14714^{*} 136167 \quad 24324624725725^{*} 236 \quad 47^{*} 573576367$}

and completion to $I$ is forced.

A subset of blocks of a $2-(v, k, \lambda)$ design will be said to be trade-free if it has no set of blocks which can be traded. It can be quickly verified that such a subset of a Fano plane will contain either fewer than four blocks, or else four blocks with every element occurring in at least one block.

Lemma 3.3. The smallest defining sets of the unique irreducible $2-(7,3,3)$ design have at least seven blocks.

Proof: As mentioned earlier there are 30 possible Fano planes on seven elements. We will identify these as $F_{i}$ and $H_{i}=(1,2) F_{i}$, for $i=1, \ldots, 15$, as in Gray [5], with $F_{1}$ being the Fano plane obtained by cycling starter block 124 modulo 7 . The remaining planes can be obtained from Table 3 which gives a permutation $\rho$ taking $F_{1}$ to $F_{i}$ for each $i=1, \ldots, 15$.

In this notation our deleted Fano planes are $F_{1}$ and $H_{14}$ respectively. It can be verified that if we let $\mathrm{F}$ be any one of the remaining planes then either

(1) $\left|F \cap\left(F_{1} \cup H_{14}\right)\right|=3$, and the remaining blocks of $F$ are trade-free, or (2) $\left|F \cap\left(F_{1} \cup H_{14}\right)\right|=1$.

There are fourteen Fano planes for which case (2) applies. Their union gives four copies of $I$ and one each of $F_{1}$ and $H_{14}$.

Now suppose $S$ is a $d_{s}(2-(7,3,3))$ defining set of $I . S$ must contain at least one block from every trade $T$ within $I$. Then $S$ must certainly contain at least two of the remaining blocks of each of the fourteen Fano planes for which case (2) applies, since $I$ contains six blocks of each such design and hence any five blocks of a Fano plane contain a trade. But each block of $S$ occurs in four of these planes so we need at least $\frac{14 \times 2}{4}=7$ blocks in $S$. 
Table 3. The Thirty Fano Planes on Seven Elements.

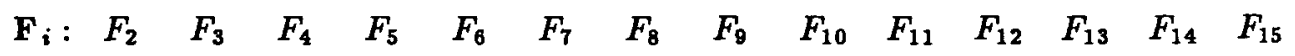

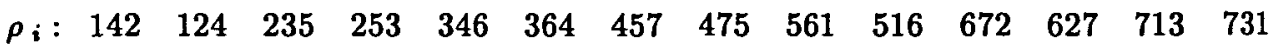

Note. For each $\mathrm{i}, F_{i}=\left(\rho_{i}\right) F_{1}$. The remaining 15 planes are given by $H_{i}=(1,2) F_{i}$.

THEOREM 3.4. The smallest defining sets of the unique irreducible $2-(7,3,3)$ design contain precisely seven blocks.

PROOF: This follows immediately from Lemmas 3.2 and 3.3.

Note that the set of blocks $S_{2}=\{123,234,345,456,567,671,712\}$ is a second nonisomorphic smallest defining set of the design $D$ containing $S_{1}$.

The automorphism groups of $S_{1}$ and $S_{2}$ have orders 2 and 14 respectively. Interestingly, $I$ can be partitioned in each case into the union of three defining sets. Examples of such partitions are: $I=S_{1} \cup(124)(365) S_{1} \cup(132645) S_{1}$ and $I=S_{2} \cup(162)(457) S_{2} \cup(126)(475) S_{2}$.

\section{Smallest defining Sets of the four $2-(8,4,3)$ Designs}

Nandi [7] showed that there are exactly four non-isomorphic $2-(8,4,3)$ designs. To assist in distinguishing these we make the following definitions: whenever the blocks of the design not containing a given element $x$ form a $2-(7,4,2)$ design we say the blocks containing $x$ are associated with respect to the associating element $x$ and these blocks are the associated blocks. It can easily be verifed that deletion of an associating element from its associated blocks leaves a $2-(7,3,1)$ design.

Examples of the four non-isomorphic $2-(8,4,3)$ designs on $\{1,2, \ldots, 7, \infty\}$ are given in Table 4. The designs can be derived from the four non-isomorphic $2-(7,3,2)$ designs given in Table 1. Thus design $\gamma^{*}$ is of type $\gamma$ derived from $C, \beta *$ is of type $\beta$ derived from $B$ and so on .

In each case the blocks have been partitioned into the associated and non-associated blocks with respect to $\infty$, so we have immediately

Lemma 4.1. Every $2-(8,4,3)$ design has an associating element. 
Table 4. The Four 2-(8,4,3) Designs and their Complements.

\begin{tabular}{|c|c|c|c|c|}
\hline \multirow{2}{*}{$\begin{array}{l}\text { Design } \\
\\
\\
\gamma^{*}\end{array}$} & \multirow{2}{*}{$\begin{array}{c}\text { Non-associated } \\
\text { Blocks } \\
2456\end{array}$} & \multirow{2}{*}{$\begin{array}{l}\text { Associated } \\
\text { Blocks } \\
713 \infty\end{array}$} & \multicolumn{2}{|c|}{$\begin{array}{l}\text { Complementary } \\
\text { design }\end{array}$} \\
\hline & & & $713 \infty$ & 2456 \\
\hline & 3567 & $124 \infty$ & $124 \infty$ & 3567 \\
\hline & 4671 & $235 \infty$ & $235 \infty$ & 1467 \\
\hline & 5712 & $346 \infty$ & $346 \infty$ & 5712 \\
\hline & 6123 & $457 \infty$ & $457 \infty$ & 6123 \\
\hline & 7234 & $561 \infty$ & $561 \infty$ & 7234 \\
\hline & 1345 & $672 \infty$ & $672 \infty$ & 1345 \\
\hline \multirow[t]{7}{*}{$\beta *$} & 2456 & $713 \infty$ & $713 \infty$ & 2456 \\
\hline & 6123 & $745 \infty$ & $745 \infty$ & 6123 \\
\hline & 1345 & $726 \infty$ & $726 \infty$ & 1345 \\
\hline & 3567 & $356 \infty$ & $124 \infty$ & 7124 \\
\hline & 4671 & $146 \infty$ & $235 \infty$ & 7235 \\
\hline & 5712 & $125 \infty$ & $346 \infty$ & 7346 \\
\hline & 7234 & $234 \infty$ & $561 \infty$ & 7156 \\
\hline \multirow[t]{7}{*}{$\alpha *$} & 1345 & $726 \infty$ & $726 \infty$ & 1345 \\
\hline & 2156 & $215 \infty$ & $734 \infty$ & 7346 \\
\hline & 2346 & $234 \infty$ & $715 \infty$ & 7156 \\
\hline & 6357 & $635 \infty$ & $124 \infty$ & 1247 \\
\hline & 6147 & $614 \infty$ & $235 \infty$ & 2357 \\
\hline & 7452 & $745 \infty$ & $136 \infty$ & 1362 \\
\hline & 7132 & $713 \infty$ & $456 \infty$ & 2456 \\
\hline \multirow[t]{7}{*}{$\delta *$} & 2456 & $245 \infty$ & $713 \infty$ & 7136 \\
\hline & 3567 & $356 \infty$ & $124 \infty$ & 1247 \\
\hline & 4671 & $467 \infty$ & $235 \infty$ & 2351 \\
\hline & 5712 & $571 \infty$ & $346 \infty$ & 3462 \\
\hline & 6123 & $216 \infty$ & $457 \infty$ & 4573 \\
\hline & 7234 & $723 \infty$ & $561 \infty$ & 5614 \\
\hline & 1345 & $134 \infty$ & $672 \infty$ & 6725 \\
\hline
\end{tabular}

Note that in Table 4 the pairs of complementary blocks are given first - these will 
have intersection type $(0,0,12,0,1)$. The remaining blocks will have type $(0,1,9,3,0)$ and each appears next to the unique block with which it has three elements in common. Table 4 also includes for later reference the designs formed by complementing each block of the given $2-(8,4,3)$ designs.

Note. Design $\gamma^{*}$ is self-complementary.

We now state without proof the following lemmas.

Lemma 4.2. A design is of type $\gamma$ if and only if every triple of elements occurs precisely once.

LEMMA 4.3. A design is of type $\beta$ if and only if it has more than one pair of complementary blocks and some triple occurring more than once. It then has three pairs of complementary blocks containing four pairs three times each, and each repeated triple occurs with a fixed pair of elements.

THEOREM 4.4. For every $2-(8,4,3)$ design $D$

$$
|d D| \geqslant 6 \text {. }
$$

Proof: By Lemma 4.1 each $2-(8,4,3)$ design $D$ can be partitioned into the associated and non-associated blocks of some element $x$. The non-associated blocks are the complementary blocks of some Fano plane and hence by Theorem 2.2 and Lemma 1.8 , a defining set must contain at least three of these blocks. The associated blocks must also contribute three blocks; otherwise, $x$ can be adjoined to some other plane than that in $D$. Six blocks in all are thus required.

A design is said to be linked if it has the property that any two blocks intersect in $\Lambda$ elements, for some constant $\Lambda$ (see, for example, Street and Street [12]).

Lemma 4.5. The associated blocks form a linked design with $\Lambda=2$.

Proof: Since, on deletion of the associating element, the associated blocks form a Fano plane with any two blocks intersecting in one element, any two associated blocks must intersect in two elements.

Lemma 4.6. The non-associated blocks form a linked design with $\Lambda=2$.

Proof: The non-associated blocks can be obtained as the complements of the blocks of a Fano plane. Since any two blocks of the Fano plane intersect in one element their complements must intersect in two.

Theorem 4.7. For every $2-(8,4,3)$ design $D$

$$
|d, D|=6 \text {. }
$$

Proof: By Theorem 4.4, it is sufficient to show that each of the designs $\gamma *, \beta *, \alpha *$ and $\delta *$ has a defining set $S$ of six blocks. 
Design $\gamma^{*}$. Suppose $S=\{2456,713 \infty, 2613,457 \infty, 2571,346 \infty\}$ is a subset of some $2-(8,4,3)$ design $D$. Since $S$ has three pairs of complementary blocks, $D$ must be of type $\gamma$ or $\beta$. By Lemma 4.3 a type $\beta$ design has only one such set of six blocks, with three of the blocks containing a common pair. Since $S$ has no such pair, $D$ is of type $\gamma$ and then, by Lemma 4.2, no triple occurs more than once. Immediately we have that $D$ must contain blocks $253 \infty, 267 \infty, 124 \infty, 7146,1345$ and 7536 , and then $561 \infty$ and 7234 .

Design $\delta *$. Take $S=\{7234,723 \infty, 1263,126 \infty, 2456,356 \infty\}$. Now by Lemma 4.1 , if $S \subset D$ for some $2-(8,4,3)$ design $D$, then $D$ can be partitioned into associated and non-associated blocks of some element. The six blocks of $S$, by Lemmas 4.5 and 4.6 , must then be able to be partitioned into the blocks of two linked designs with $\Lambda=2$. Since 7234 and 1263 cannot occur with $126 \infty$ the partition must be

\section{$\begin{array}{llllllll}1263 & 7234 & 2456 & \ldots & 126 \infty & 356 \infty & 723 \infty\end{array}$.}

The elements common to either side are 2 on the left and $\infty$ on the right, but since 2 also appears on the right, $\infty$ must be the associating element. Further, the blocks on each side lead uniquely to the associated and non-associated blocks respectively, and can be completed in only one way, giving design $\delta *$.

Design $\beta_{*}$. Take $S=\{713 \infty, 2456,726 \infty, 1345,2347,234 \infty\}$. By Lemma 4.3, the design must be of type $\beta$ and consideration of block intersections gives that the remaining pair of complementary blocks is $745 \infty$ and 1236 . Also, by the same Lemma, since the triple 234 is repeated, repeated triples must occur with 7 and $\infty$. Now element 1 must occur with each of 7 and $\infty$ twice more and pair 13 must not occur again so we must have blocks with form

\section{$71 a b \quad \infty 1 a b$ 71cd $\infty 1 c d$ 73ef $\infty 3 e f$.}

5 and 6 must each occur again twice with 1 and 3, with the pair 56 only occurring twice more and so we have

$$
\begin{array}{llllll}
716 a & \infty 16 a & 715 b & \infty 15 b & 7356 & \infty 356
\end{array}
$$

and the design completes to $\beta *$.

Design $\alpha *$. Take $S=\{1345,726 \infty, 2156,215 \infty, 3567,356 \infty\}$. As in case (ii) we attempt to partition $S$ into linked designs. This can be done in two ways.

$$
\operatorname{Case}(A)
$$

2156

7356

1345
$215 \infty$

$356 \infty$

$726 \infty$
$\operatorname{Case}(B)$

2156

$215 \infty$

7356

$356 \infty$

$726 \infty$ 
In case $(B), 6$ is the common element on the left but also appears on the right, while the common element 5 on the right appears also on the left. Hence no element can be an associating element for this partition. In case $(A)$ we similarly see that only $\infty$ can be the associating element and in this case we must complete our associated and non-associated blocks uniquely to produce design $\alpha *$.

\section{Smallest Defining Sets of 3-designs}

Suppose $T$ is a $3-(v, k, \lambda)$ design. Consider the set of blocks containing any given element $x$, with $x$ deleted. Since $T$ is a 3-design, this set of blocks must be a 2-design, with each pair occurring $\lambda$ times. Such a design is called a restriction (on $x$ ). For any set of blocks $S=\left\{b_{i}\right\}$, let $S(\infty)=\left\{b_{i} \cup\{\infty\}\right\}$ for some additional element $\infty$. Then the following result is well known and stated without proof (see Hughes and Piper [6])

Lemma 5.1. Suppose $D$ is a $2-(2 n+1, n, \lambda)$ design. Then $D(\infty) \cup \widetilde{D(\infty)}$ is a $3-(2 n+2, n+1, \lambda)$ design.

We will call such a design an extension by complementation of $D$; see Breach [3] for further discussion of extensions to 3-designs. The following Lemma is also clear.

LEмma 5.2. Suppose $T$ is an extension by complementation of some 2-design $D$ for some element. Then $T$ is also an extension by complementation of the restriction of $T$ on $x$, for every element $x$ of the underlying set of $T$.

ProOF: Since $T$ is an extension by complementation the blocks can be arranged in complementary pairs. For any element $x, T$ must then be the union of the blocks containing $x$ together with their complements.

Theorem 5.3. Suppose $S$ is a $d(2-(2 n+1, n, \lambda))$ defining set. Then $S(\infty)$ is a $d(3-(2 n+2, n+1, \lambda))$ defining set, provided all 3-designs with the required parameters can be obtained by extension by complementation.

Proof: Suppose $S$ is a $d D$ defining set for some $2-(2 n+1, n, \lambda)$ design $D$. Further suppose $S(\infty) \subseteq T$ for some $3-(2 n+2, n+1, \lambda)$ design $T$, where $T$ is obtained by extension by complementation. By Lemma 5.2, $T$ must be able to be obtained by extension by complementation of the restriction of $T$ on $\infty, \mathcal{D}$ say. Then we have $S(\infty) \subseteq \mathcal{D}(\infty)$ and since $S$ is a defining set, $\mathcal{D}=D$; that is, $D(\infty) \subseteq T$. But $T$ must contain the complement of each block and so $T=D(\infty) \cup \widetilde{D(\infty)}$. The 3-design containing $S(\infty)$ is then unique, and thus $S(\infty)$ is a defining set as required.

THEOREM 5.4. Suppose, for some given values of $n$ and $\lambda$, every $3-(2 n+2, n+1, \lambda)$ design is obtainable by extension by complementation. Then each $d(3-(2 n+2, n+1, \lambda))$ defining set gives rise to a $d(2-(2 n+1, n, \lambda))$ defining set with equal cardinality. 
Proof: Suppose the given conditions hold and $S$ is a $d(3-(2 n+2, n+1, \lambda))$ defining set of some 3-design $D=X(\infty) \cup \widetilde{X(\infty)}$, where $X$ is a $2-(2 n+1, n, \lambda)$ design. Then for each block $b_{i} \in S$ define:

$$
c_{i}= \begin{cases}b_{i}, & \text { if } \infty \in b_{i} \\ \tilde{b}_{i}, & \text { otherwise }\end{cases}
$$

In either case $\infty \in c_{i}$ for all $i$. Let $\mathcal{C}$ be the set of blocks $\left\{c_{i}\right\}$ with the element $\infty$ deleted. Clearly $\mathcal{C} \subseteq X$ and $|\mathcal{C}|=|S|$. If $\mathcal{C}$ is not a $d(2-(2 n+2, n+1, \lambda))$ defining set then $\mathcal{C}$ must belong to a $2-(2 n+1, n+1, \lambda)$ defining set $X^{\prime}$, distinct from $X$. Then $D=X(\infty) \cup \widehat{X(\infty)}$ and $D^{\prime}=X^{\prime}(\infty) \cup \widehat{X^{\prime}(\infty)}$ are both 3-designs containing $S$, contradicting the assumption that $S$ is a defining set. Hence $\mathcal{C}$ must be a defining set of the required form.

As a consequence of Theorems 5.3 and 5.4 we have

TheOREM 5.5 . If $D$ is a $3-(2 n+2, n+1, \lambda)$ design, necessarily obtainable by extension by complementation, and $D^{x}$ is its restriction on $x$, then

$$
|d, D|=\left|d_{s} D^{x}\right|
$$

where the parameters of the defining sets correspond to those of the respective 2-design and 3-design.

Corollary 5.6. If $D$ is a $3-(8,4,1)$ design then

$$
\left|d_{s} D\right|=3 \text {. }
$$

Proof: This follows from Theorem 2.2, since a 3-(8,4,1) design is unique up to isomorphism and obtainable by extension by complementation of a Fano plane. For example, we give the design $\mathcal{F}$ with blocks

$\begin{array}{lllllll}124 \infty & 235 \infty & 346 \infty & 457 \infty & 561 \infty & 672 \infty & 713 \infty \\ 3567 & 1467 & 1257 & 1236 & 2347 & 1345 & 2456 .\end{array}$

The restriction of $\mathcal{F}$ on $\infty$ has a typical defining set $\{124,235,346\}$ corresponding to the $3-(8,4,1)$ defining set $\{124 \infty, 235 \infty, 346 \infty\}$.

Corollary 5.7. If $D$ is a $3-(8,4,2)$ design then

$$
|d D|=6 .
$$

This follows from Theorem 2.4 and since all designs with the given parameters are obtainable by extension by complementation (see Nandi [9]). 
Corollary 5.8. If $D$ is a $3-(8,4,3)$ design then

$$
|d, D|=9 \text { or }|d, D|=7 \text {. }
$$

Proof: This follows from Theorems 3.1 and 3.4 and since the ten $3-(7,3,3)$ designs are easily seen to be obtained from the ten $2-(7,3,3)$ designs by extension by complementation (see Morgan [8]).

\section{ACKNOWLEDGEMENTS}

I would like to thank Professor Anne Penfold Street for her assistance in the preparation of this paper. The work presented in this paper has been sponsored by the Australian Research Grants Scheme and by a University of Queensland special projects grant. Many of the computations were carried out using software packages Cayley [4] and nauty [7] on the Pyramid 9810 machine of the Department of Mathematics, The University of Queensland.

\section{REFERENCES}

[1] N.L.Biggs and A.T.White, Permutation Groups and Combinatorial Structures (Cambridge University Press, Cambridge, 1979).

[2] Elizabeth J. Billington, 'Further Constructions of Irreducible Designs', Congressus $\mathrm{Nu}$ merantium 35 (1982), 77-89.

[3] D.R.Breach, 'The $2-(9,4,3)$ and $3-(10,5,3)$ Designs', Combin. Theory, Ser. A 27 (1979), 50-63.

[4] John Cannon, A Language for Group Theory (Department of Pure Mathematics, University of Sydney, 1987).

[5] Ken Gray, 'Designs Carried by a Code', Ars Combin. 23B (1987), 257-271.

[6] D.R.Hughes and F.C.Piper, Design Theory (Cambridge University Press, Cambridge, 1985).

[7] Brendan D. McKay, nauty User's Guide (Version 1.2) (Australian National University Computer Science Technical Report TR-CS-87-03, 1987).

[8] Elizabeth J. Morgan, 'Some small quasi-multiple designs', Ars Combin. 3 (1977), 233-250.

[9] Harikinkar Nandi, 'A further note on non-isomorphic solutions of incomplete block designs', Sankhya 7 (1945-46), 313-316.

[10] C.A.Rodger, 'Triple Systems with a Fixed Number of Repeated Blocks', J. A ustral. Math. Soc. (A) 41 (1986), 180-187.

[11] R.G.Stanton and R.J.Collens, 'A computer system for research on the family classification of BLBDs', Proc.Internat.Congress on Combinatorial Theory, Accad. dei Lincei (Rome, 1973), I (1976), 133-169.

[12] Anne Penfold Street and Deborah J.Street, Combinatorics of Experimental Design (Clarendon Press, Oxford, 1987). 
[13] W.D.Wallis, private communication; see Morgan [8].

Department of Mathematics

University of Queensland

St Lucia Qld 4067 Australia 